\title{
Cloud-based Assisted Decision Making Scheme for Electric Power Dispatch System
}

\author{
Bingquan Zhu ${ }^{1}$, Zhongming Xiang ${ }^{1}$, Qifeng $\mathrm{Xu}^{1}$, Yajie Tang ${ }^{2, *}$, Cuiyang Wang ${ }^{2}$ and Jian Tang $^{3}$ \\ ${ }^{1}$ Power Dispatch and Communication Center, State Grid Zhejiang Electric Power Company, Hangzhou, 310007, Zhejiang, China \\ ${ }^{2}$ College of Electrical Engineering, Zhejiang University, Hangzhou, 310027, Zhejiang, China \\ ${ }^{3}$ Hangzhou Power Supply Branch, State Grid Zhejiang Electric Power Company, Hangzhou, 310009, Zhejiang, China \\ ${ }^{*}$ Corresponding author
}

\begin{abstract}
Cloud computing is one of the key enablers for modern electric power dispatch systems, in which many assisted decision making applications are involved. This work focuses on one important decision making scenario, i.e., providing suggested actions for alarming signals from electric component in both transmission and distribution network. Knowledge base is set up to find template to characterize the alarming signal patterns. A real-time template matching scheme is then established to find out the best candidate to aware current fault situation. The effectiveness of the proposed approach is verified using realworld electric component alarming data. The results indicate that the workload of operator is significantly reduced and the assisted decision accuracy is improved.
\end{abstract}

Keywords-cloud computing; data mining; decision making; electric power dispatch center; service-oriented architecture

\section{INTRODUCTION}

Cloud computing is a revolutionary technique which is able to significantly boost both computing and storage capability of a given information system [1]. Electric power dispatch system is one of the most complicated, wide-area, real-time system in the world [2]. The recent development of power dispatch system is enabled by the integration of cloud computing technology. Therefore, there is a strong need to reinvent state-of-the-art energy management system (EMS) applications in a cloud environment, for better performance and scalability. This need is particularly emphasized in the vision of smart grid [3].

Decision making is a major role in power dispatch system. However, existing practice is commonly based on the justification of human, i.e., the operators with professional knowledge. With the increase the electric power system scale, renewable integration and complex system disturbances, it is difficult for experienced operators to identify potential risk and aware fast-evolving situation [4]. An intelligent, automatic, decision support scheme is expected to assist operator to find out what is happening given a huge stream of alarming signals from various electric component in both transmission and distribution network in real time.

As the alarming signal data is highly unstructured, especially for the ones in non-English speaking countries like China, this assisted decision making intelligence is extremely difficult to design and implement. Power engineering community has been working on this topic for years.
Reference [5] proposed a knowledge-based intelligent energy management for building automation system. Reference [6] developed a decision-making system for accident operation for a local urban power grid. These existing attempts failed to utilize the computing capability and information availability in modern power dispatch center. To solve this problem, this work introduces data mining [7] method to process alarming signals and extract knowledge from them, so as to establish intelligent system and enhance operation performance.

\section{Cloud-BASEd Electric POWER DisPatch SySTEM}

To fulfill the requirement of intelligent assisted decisionmaking scheme and make the most benefit of cloud computing, the proposed system is designed following the concept of service oriented architecture (SOA) [8], which is focused on the following features: intellective, interconnect and re-usable. It enables us to protect the investment in software development, prolong its life cycle and reuse its components.

The framework of the proposed cloud-based power dispatch system is demonstrated in Fig. 1, where three layers are observed from bottom to top, namely, infrastructure layer, service application layer and service access layer.

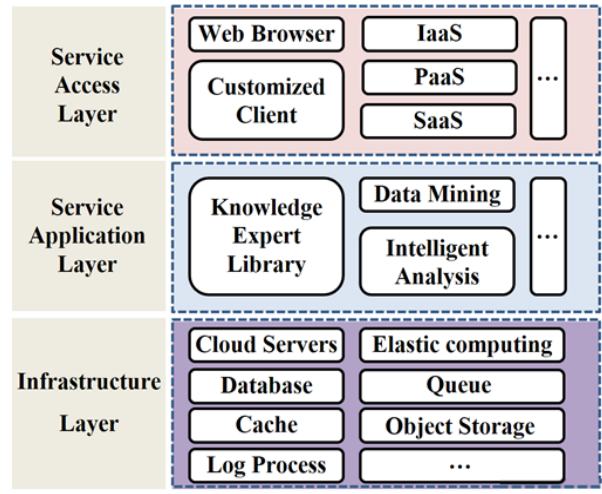

FigURE I. FrAmEWORK OF A CLOUd-BASED POWER DiSPATCH SYSTEM

1) Infrastructure Layer: Through the combination of virtualized hardware and related management software resources, infrastructure layer offers dynamic and agile fundamental services, for instant message dispatch, log processing, on-line analytics and cloud monitoring. 
2) Service Application Layer: In this layer, the cloud-based packaged services consist of data acquisition and data switching platform. The system deploys multiple intelligent analysis services, with various data mining algorithms, especially for the assistant decision-making scheme.

3) Service Access Layer: As a new business model, cloud computing presents itself as IT service to every user. Infrastructure as a Service (IaaS), Platform as a Service (PaaS) and Software as a Service (SaaS), those are 3 types of providing cloud computing service.

\section{Assisted DeCision MAKING SCHEME}

\section{A. Basic Idea}

Smart and refined operation is the development trend of modern power system dispatch and communication centers, in which many assisted decision making schemes are involved.

This work focuses on determining the solving actions in response to alarm signals from transmission and distribution components, e.g., circuit breakers, transformers, switches, and various auxiliary sub-systems. These signals are, if possible, difficult to model and represent, as they are in the form of small phrases or sentence, including the name of alarming component, its location, time, action, as well as a series of adjective expression. Basically there is no standard communication protocol available so far, vendors of these electric components simply use plain text to report abnormality and incident. For system operator, once a fault occurs, he or she has to address a significant number of alarming messages sent from electric components via message bus of the cloud based power dispatch system. These messages may pop up very rapidly and make it extremely misleading in situation awareness, especially during a severe fault.

Therefore, an urgent need for an assisted decision making tool for operators is observed. It is expected to identify the root reason or major signal from massive alarming messages and offer suggested solving actions according to current situation. Such intelligence was believed to be impossible before, because of data availability and limited computing resource. Enabled by cloud based architecture of modern power system dispatch system shown in Section II, it is now technically feasible to develop an intelligent system to process online alarming signals and offer assisted decision making capability, which is exactly the purpose of this work.

\section{B. Ontology-based Knowledge Base}

In order to establish the intelligent system aforementioned, one has to setup knowledge base to store, manage and utilize existing professional experience and use cases.

Building knowledge base has been a hot spot in database research area, and also evidently a critical part in power system dispatch, due to its strong influence on the efficiency of data storage, maintenance, retrieval and reasoning. Considering the unique features of cloud-based power dispatch system, the proposed system chooses an ontology-based representation method to build knowledge base.
Knowledge entries are arranged in the form of a tree-like hierarchical structure with multiple links, shown in Fig. 2 as an example. Through a continuous filling of the content in this structure, the knowledge base will be enriched and improved.

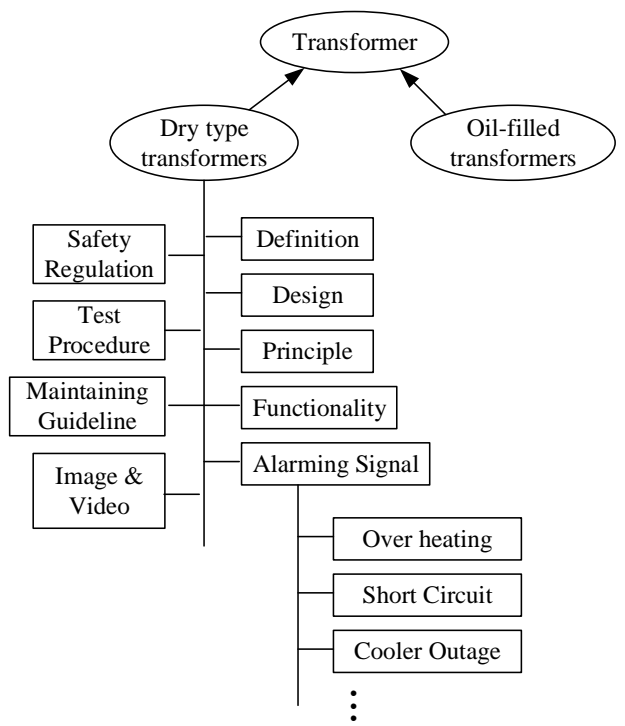

FIGURE II. TRANSFORMER KNOWLEDGE POINT HIERARCHY DIAGRAM

\section{Smart Action Suggestion for Alarming Signals}

Smart action suggestion for alarming signals is the goal of this research. Such assisted decision-making scheme integrates data mining, automatic inference and human guidance.

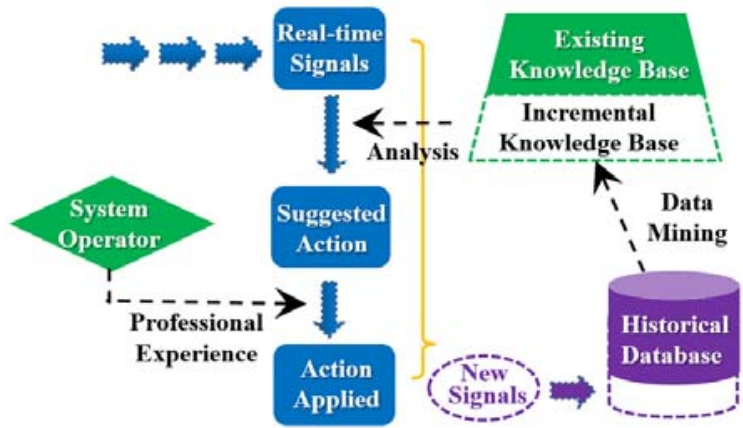

FIGURE III. FLOWCHART OF THE ASSISTED DECISION MAKING SCHEME

Fig. 3 illustrates the idea of smart action suggestion for alarming signals. Its detailed procedure is described as follows.

First, as a preparation, one has to setup a reference knowledge base, based on existing power dispatch manual or guideline, which offer standard suggested actions for typical alarming signals. Such information is provided by the ontology-based method in the sub-section above. Based on the existing knowledge base, we are ready to go over the flowchart of the smart action suggestion procedures.

As the existing daily practice, once there is one or more alarming signals, such messages will be displayed on the operator screen in real time. Based on his/her professional experience, the operator has to come up with the solving action for the alarming signal manually, in a timely manner. 
With the help of the proposed smart decision making scheme, before going to the operator, the real-time alarming signals are firstly analyzed and compared to existing knowledge base, in order to see if a sufficiently similar alarming signal set (it is called template in the following discussion) has been recorded. If yes, a suggested action is prepared for the reference of the operator according to exist experience from the knowledge base. In this case, both time and energy are saved for the operator to come up with the solving actions. As this process is automatically performed, it is able to significantly increase the working efficiency and correctness of solving actions of alarming signals.

Also, for the case that is unknown to existing knowledge base, the signals and applied actions are then stored in a historical database. Such record entries are learned via data mining, an extra knowledge base is setup to store the result from the incremental learning. The resultant knowledge base is able to work seamlessly with existing knowledge base, so as to offer more accurate template matching capability.

Note that, this scheme provides the opportunity to bring offline intelligence to online decision, which is a huge progress in smart grid development. Furthermore, the cloudbased system framework can continually scale its performance through the accumulation of data, by cloud expansion.

As time pass by, with the accumulated signals and feedback, the overall knowledge base is able to continuously develop itself. To add up, the knowledge base could give out suggested action towards single signals, or a comprehensive analysis result for several signals in a period. For instance, when there is a fault, the system will receive a burst of fault signals as well as some noise signals. At this time, the proposed assistant decision-making scheme classifies those signals by using template matching, and provides suggested action for the certain fault. Obviously, it reduces the load of manual decision-making jobs for the operators. Also, it improves the accuracy in dealing with those faults.

\section{SOA Packaging}

The developed assisted decision making scheme is packaged using SOA technology, which offers a unified and consistent interface to external system modules or various use cases. As a packaged component model, it is able to integrate the functions of the system. Released services can interact through specified interfaces and achieve modularization management, improving the operation efficiency of the system.

By means of normalized message and service bus, the system is able to apply different services in a module setup. In addition, the service of assisted decision-making scheme is in the most upper level of application layers, thus every scheme algorithm could re-use the basic services as much as possible.

Note that, this design achieves economics of scale in implementing the system software incrementally. SOA based power dispatch system can grow and change quickly, integrate and offer new feature more flexibly, because it can add and remove components from its SOA architecture on demand.

\section{E. Implementation with Big Data}

Power dispatch system gains a rich supply of various kinds of data. The processing system not only needs to deal with structured data like real-time measuring data from networked sensors or alarm data from electric components, but also unstructured data like operation log, image, etc. Traditional local relational database could barely meet the demand of processing such data in an efficient and scalable way.

As one of the most important advantages brought by cloud computing, distributed storage and processing system is able to aforementioned problem. The widely used HDFS (Hadoop Distributed File System) in cloud computing is the basis of distributed storage. Besides, HBase (Hadoop Database) is a real-time database built up from HDFS, possessing promising features, such as strong fault tolerance, high reliability, high availability, high throughput, etc. These main characteristics bring convenience to process streaming data and oversized files. OSS (Open Storage Service) is a cloud storage openresource interface. Given any time, location or equipment, users are able to upload and download data through a simple interface, and are also able to use web page to manage data. By using OSS, the implementation scheme could properly deal with unstructured data in power dispatch system.

Note that, a local fault in a distributed database group does not affect the processing capability of other instances, making the system more stable and robust. The separation of the backstage database and monitoring platform leads to a coarsegrained and fault-resistant design.

\section{CASE STUdY}

\section{A. Application Setup}

The knowledge base is the logical process part of the scheme. Considering the characteristics of power dispatch system as well as the ones of cloud computing technology, the designed knowledge base realizes resource virtualization with the help of cluster based servers. Using the redundant distributed storage system and data management model based on the column storage, the reliability of massive state data for decision making application has been ensured. In addition, a parallel processing system based on Map Reduce programming model offers high performance computing ability and generalized parallel algorithm development environment.

The studied assisted decision-making scheme, a.k.a. smart action suggestion for alarming signals, is implemented with knowledge base for knowledge search, data mining and decision making. Operator can acquire assistant disposal for daily real-time faults, provided with effective signal analysis and decision support information. Each algorithm can be viewed as a plug and play module. It is feasible to extend to other advanced data mining algorithm or novel features. Thus it enhances the overall performance of electric power dispatch.

In data mining part of assisted decision-making scheme, the input sources cover all of historical signals. In the latter real-time analysis part, a scrolling queue is used to process 
continuous signals. Therefore, the algorithm is able to deal with a section of signals caused by accident on the spot.

\section{B. Suggested Action Results}

As a case study, we use a clip of real world alarming signal stream from electric power dispatch and communication center of State Grid Zhejiang Electric Power Company. The raw alarming data of two typical faults is shown in Fig. 4.

Following the rule of establishing knowledge base, Fig. 5 shows a clustered typical diagnosis module through data mining. It is able to characterize the faults with similar alarming signal pattern.

\begin{tabular}{|c|c|c|}
\hline Time Stamp & $\begin{array}{l}\text { Transforme } \\
\text { Substation }\end{array}$ & Signal Content \\
\hline $17 / 08 / 0416: 52: 34$ & QiaoLin & 220kV.QiaoWan2411Line Switch / Turned Off \\
\hline $17 / 08 / 0416: 52: 34$ & QiaoLin & QiaoWan2411Line Second Set of Protection/ Reset \\
\hline 17/08/04 16:52:34 & QiaoLin & QiaoWan2411Line Interval Fault Alarm / Action \\
\hline 2017/08/04 16:52:34 & QiaoLin & 220kV.QiaoWan2411Line Switch / Turned Off \\
\hline 2017/08/04 16:52:34 & QiaoLin & Integral Station Fault Alarm/ Action \\
\hline 2017/08/04 16:52:34 & QiaoLin & QiaoWan2411Line Phase-B of Switch / Turned Off \\
\hline 2017/08/04 16:52:34 & QiaoLin & Security Alarm / Action \\
\hline 2017/08/04 16:52:34 & QiaoLin & QiaoWan2411Line First Set of Pro \\
\hline 2017/08/04 16:52:34 & QiaoLin & QiaoWan2411Line Second Set of 1 \\
\hline 2017/08/04 16:52:35 & QiaoLin & 220kV.QiaoWan2411Line Switch/ \\
\hline $2017 / 08 / 041$ & QiaoLin & QiaoWan2411Line Phase-B of Switch / Turned On \\
\hline 2017/08/04 1 & QiaoLin & QiaoWan2411Line Interval Fat \\
\hline 2017/08/04 16:52:35 & oLin & Integral Station Fault Alarm / Reset \\
\hline $2017 / 08 / 0416: 52: 35$ & QiaoLin & QiaoWan2411Line Protection Reclosing / Action \\
\hline 2017/08/04 16:52:35 & QiaoLin & Security Alarm / Reset \\
\hline $2017 / 0$ & & 26 \\
\hline $2017 / 08 / 1717: 57: 21$ & QiaoLin & FuQiao2401Line Second Set of Protection / Reset \\
\hline 2017/08/17 17:57:21 & QiaoLin & FuQiao2401Line Interval Fault Alarm / Action \\
\hline 2017/08/17 17:57:21 & QiaoLin & 220kV. FuQiao2401Line Switch / Turned Off \\
\hline 2017/08/17 17:57:21 & QiaoLin & Integral Station Fault Alarm / Action \\
\hline 2017/08/17 17:57:21 & QiaoLin & FuQiao2401Line Phase-A of Switch / Turned Off \\
\hline $2017 / 08 / 17 \quad 17: 57: 21$ & QiaoLin & Security Alarm / Action \\
\hline 2017/08/17 17:57:21 & QiaoLin & FuQiao2401Line First Set of Prote \\
\hline 2017/08/17 17:57:21 & QiaoLin & FuQiao2401Line Second Set of Protection / Action \\
\hline $2017 / 08 / 1717: 57: 22$ & QiaoLin & 220kV. FuQiao2401Line Switch / Turned On \\
\hline 2017/08/17 17:57:22 & QiaoLin & FuQiao2401Line Phase-A of Switch / Turned On \\
\hline $2017 / 08 / 17 \quad 17: 57: 22$ & QiaoLin & FuQiao2401Line Interval Fault Alarm / Reset \\
\hline 2017/08/17 17:57:22 & QiaoLin & Integral Station Fault Alarm/ Reset \\
\hline 2017/08/17 17:57:22 & QiaoLin & FuQiao2401Line Protection Reclosing / A \\
\hline $2017 / 08 / 1717: 57: 41$ & QiaoLin & Security Alarm / Reset \\
\hline
\end{tabular}

FIGURE IV. TWO SETS OF ALARMING SIGNAL DATA

\begin{tabular}{l} 
Time Line Voltage Degree \\
\begin{tabular}{lll}
$220 \mathrm{kV}$ & $\begin{array}{l}\text { Feature Words } \\
\text { Line Switch }\end{array}$ & $\begin{array}{l}\text { Action } \\
\text { Tumed off }\end{array}$ \\
\hline & Second Set of Protection Action & Reset \\
\hline $220 \mathrm{kV}$ & Interval Fault Alarm & Action \\
\hline & Line Switch & Tumed off \\
\hline $220 \mathrm{kV}$ & Integral Station Fault Alarm & Action \\
\hline & Phase of Switch & Tumed off \\
\hline & Security Alarm & Action \\
\hline $220 \mathrm{kV}$ & First Set of Protection Action & Action \\
\hline $22 \mathrm{kV}$ & Second Set of Protection Action & Action \\
\hline & Switch & Turned on \\
\hline & Phase of Switch & Tumed on \\
\hline & Interval Fault Alarm & Reset \\
\hline & Integral Station Fault Alarm & Reset \\
\hline & Line Protection Reclosing & Action \\
\hline & Security Alarm & Reset
\end{tabular} \\
\hline
\end{tabular}

FIGURE V. THE TEMPLATE OBTAINED BY DATA MINING

Based on the obtained templates, real-time alarming signals can be analyzed as shown in Fig. 6. As we can observe, it realizes multiple signals classification through the combination of accident signals and noise signals. On the one hand, the assisted decision making scheme provides a suggested action, which effectively improves the efficiency of operator's justification. On the other hand, it also promotes the normalization and standardization of decision-making process in a modern power dispatch center.

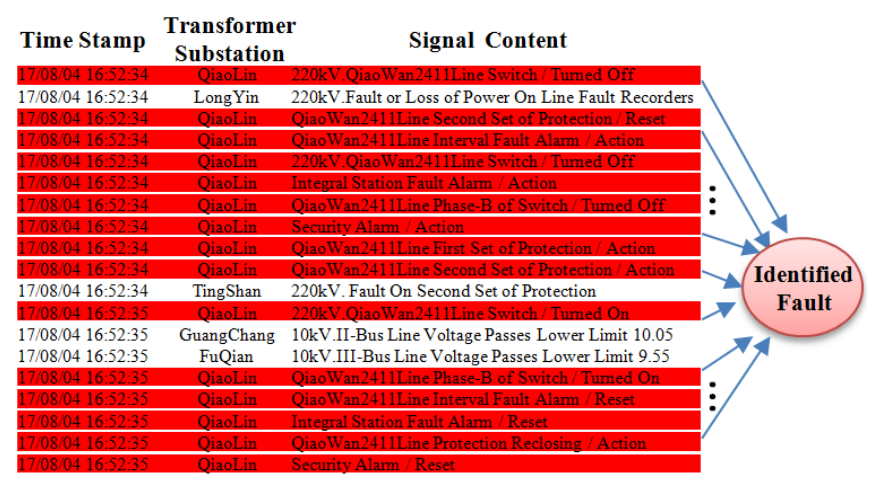

FIGURE VI. RESULTS OF REAL-TIME ALARMING SIGNAL ANALYSIS

\section{CONCLUSION}

A cloud-based assisted decision making scheme for online solving action suggestion of alarming signal is proposed, designed and implemented in this work. Packaged by SOA technology, it is able to offer a unified interface for maximum re-use. The proposed data mining based action suggestion method is adaptive to various alarming signal pattern and effective in incremental learning new fault types for better decision making performance.

\section{ACKNOWLEDGEMENT}

This research is supported by Science and Technology Project of State Grid Zhejiang Electric Power Company (5211HZ15018Y).

\section{REFERENCES}

[1] J. Singh, J. Powles, T. Pasquier, J. Bacon, "Data Flow Management and Compliance in Cloud Computing". IEEE Cloud Computing. vol. 2, no. 4, pp. 24-32, July 2015.

[2] F. Maghsoodlou, R. Masiello and T. Ray, "Energy management systems," IEEE Power and Energy Magazine, vol. 2, no. 5, pp. 49-57, Sept.-Oct. 2004.

[3] H. Farhangi, "The path of the smart grid," IEEE Power and Energy Magazine, vol. 8, no. 1, pp. 18-28, Jan. 2010.

[4] M. M. Kokar and M. R. Endsley, "Situation Awareness and Cognitive Modeling," IEEE Intelligent Systems, vol. 27, no. 3, pp. 91-96, MayJune 2012.

[5] H. Wicaksono, S. Rogalski and E. Kusnady, "Knowledge-based intelligent energy management using building automation system," Proceedings IPEC 2010 Conference, Singapore, 2010, pp. 1140-1145..

[6] X. Zhang, J. Li, C. Jiang, B. Liu and A. Yang, "Aided decision-making system of accident operation and command for local power grid in Changchun," 2011 IEEE International Conference on Automation and Logistics (ICAL), Chongqing, 2011, pp. 442-445.

[7] M.-S. Chen, J. Han and P. S. Yu, "Data mining: an overview from a database perspective," IEEE Transactions on Knowledge and Data Engineering, vol. 8, no. 6, pp. 866-883, Dec 1996.

[8] M. Valipour, B. AmirZafari, K. Maleki, N. Daneshpour. "A brief survey of software architecture concepts and service oriented architecture". 2nd 
IEEE International Conference on Computer Science and Information

Technology, 2009, pp. 34-38. 